\title{
Paolo Zaccone*
}

Università degli Studi di Torino

\section{RIFORMA COSTITUZIONALE RENZI-BOSCHI}

DOI: http://dx.doi.org/10.12775/TSP-W.2017.004

Data wpływu: 13.02.2017

Data akceptacji: 04.06.2017

Italian constitutional reform Renzi-Boschi. The essay the potential application of the constitutional reform Renzi-Boschi. If the reform will be approved, the balance of powers will be redesigned. In the new Constitution, only the Chamber of the Deputies will confer a vote confidence on the government (until now, the government has had to obtain the confidence of both the Chamber and the Senate). The new Senate of the Republic will no longer be elected by the citizens, but rather selected by the regional councils from among their members and some mayors, in order to represent "territorial institutions."

Keywords: constitutional reform; Italy; Senate; Renzi-Boschi.

Reforma konstytucyjna Renzi-Boschi. W artykule przedstawione zostały podstawowe założenia tzw. reformy konstytucyjnej Renzi-Boschi. Reforma przewidywała m.in odejście od równorzędnej dwuizbowości, redukcję składu osobowego Senatu oraz zmienię jego funkcji ustrojowych. Ponadto, zakładała zmianę podziału administracyjnego kraju. Pomimo odrzucenia reformy w referendum ogólnokrajowym, warto przybliżyć założenia i uwarunkowania polityczno-społeczne, które jej towarzyszyły.

Słowa kluczowe: reforma konstytucyjna; Republika Włoska; Senat; Renzi-Boschi.

* Paolo Zaccone - dottore in giurisprudenza, Università degli Studi di Torino. 


\section{PREMESSA}

Dopo mesi di dibattiti, discussioni e forte tensione politica, il 4 dicembre 2016 i cittadini italiani si sono recati alle urne per pronunciarsi sulla riforma della Costituzione approvata dal Parlamento con legge costituzionale n. 88 del 15 aprile 2016. La legge di revisione costituzionale si proponeva in particolare di modificare 1'assetto istituzionale dell'ordinamento italiano: la finalità principale consisteva nel superamento del bicameralismo perfetto in modo da razionalizzare la forma di governo parlamentare e garantire la stabilità dell 'esecutivo. Le prime discussioni sull'opportunità del bicameralismo paritario si manifestarono fin dal periodo immediatamente successivo all 'entrata in vigore della Costituzione ${ }^{1}$.

Successivamente, a partire dall'inizio degli anni ,80, emerse la volontà di rafforzare l'efficienza e la stabilità dell 'esecutivo. Pertanto fu istituita una Commissione parlamentare per le riforme costituzionali, detta Commissione Bicamerale, con 1'obiettivo di modificare 1'ordinamento costituzionale, ossia 1'organizzazione e i rapporti tra i poteri e le istituzioni repubblicane. A seguito del fallimento di tale tentativo, negli anni ,90 furono costituite due diverse Commissioni Bicamerali. Tuttavia i progetti di riforma elaborati all'interno delle Bicamerali non ebbero fortuna a causa di contrasti politici tra le diverse forze. Nel 2006 la coalizione di centrodestra propose una legge di revisione costituzionale. Questa fu approvata in sede parlamentare a maggioranza assoluta, ma venne poi respinta a seguito della consultazione referendaria.

La stessa sorte è recentemente toccata alla proposta di riforma costituzionale avanzata dal governo Renzi. L'8 aprile 2014 venne presentato un disegno di legge costituzionale di iniziativa governativa. Al testo furono apportate modifiche sia dal Senato sia dalla Camera dei Deputati.

Successivamente, il 13 ottobre 2015, il Senato approvò il disegno di legge apportando ulteriori modifiche. La Camera approvò il nuovo testo in data 11 gennaio 2016.

Il Senato approvò il medesimo in seconda deliberazione in data 20 gennaio 2016, con 180 voti favorevoli, 112 contrari e un astenuto. Infine la Camera lo approvò definitivamente il 12 aprile 2016, con 361 voti favorevoli, 7 contrari e 2 astenuti. In questa occasione le opposizioni abbandonarono l'aula in segno di dissenso. La legge di revisione costituzionale venne pubblicata sulla Gazzetta Ufficiale il giorno 15 aprile 2016, Serie Generale numero 88.

${ }^{1}$ F. Occhetta, La riforma della Costituzione, www.stefanoceccanti.wordpress.com. (12.06.2017). 
Il 20 aprile un gruppo di deputati e di senatori presentò una richiesta di referendum costituzionale $\mathrm{e}$, in seguito ad una procedura di raccolta firme, fu altresì presentata una richiesta di referendum popolare.

Il Consiglio dei Ministri fissò la data del referendum al 4 dicembre 2016.

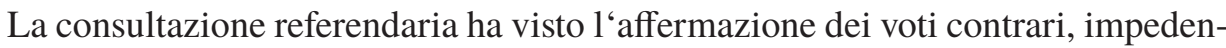
do perciò l'entrata in vigore della riforma. L'iter di approvazione delle leggi di revisione della Costituzione italiana è previsto dall 'art. 138 della Costituzione. Questo infatti stabilisce che le leggi di revisione costituzionale e le altre leggi costituzionali devono essere adottate da ciascuna Camera con due successive deliberazioni ad intervallo non minore di tre mesi. Nella seconda votazione le suddette leggi devono essere approvate a maggioranza assoluta dei componenti di ciascuna Camera. In questo caso, tuttavia, queste vengono sottoposte a referendum popolare qualora ne facciano richiesta un quinto dei membri di una Camera o cinquecentomila elettori o cinque consigli regionali, entro tre mesi dalla loro pubblicazione.

Se invece nella seconda votazione vengono approvate a maggioranza qualificata di due terzi dei componenti di ciascuna Camera, non si fa luogo a referendum e tali norme vengono promulgate immediatamente. Il referendum costituzionale è confermativo: affinché il referendum sia valido non è previsto il raggiungimento di alcun quorum; la legge viene promulgata se i voti favorevoli superano quelli sfavorevoli.

Il risultato della consultazione referendaria dello scorso 4 dicembre è stato sfavorevole all'approvazione della legge di revisione costituzionale. L'affluenza alle urne si è attestata al 65,47 \% degli aventi diritto al voto, con la netta affermazione dei voti contrari alla riforma, pari al 59,12 \% di voti validi contro il 40,88 $\%$ di voti favorevoli. La legge di revisione costituzionale n. 88 del 15/04/2016 modificava il titolo IV della parte I e i titoli I, II, III, V, VI della parte II della Costituzione, intervenendo su 47 articoli dei 139 presenti in Costituzione. Tuttavia alcuni di questi venivano modificati marginalmente, conseguentemente all'abolizione delle province e del bicameralismo perfetto.

La riforma prevedeva innanzitutto il superamento del bicameralismo perfetto attraverso una riforma strutturale e funzionale del Senato. Modificava inoltre il procedimento di formazione legislativa e la disciplina del referendum. Aboliva il CNEL (Consiglio nazionale dell 'economia e del lavoro) e le province e modificava il titolo V relativo agli enti locali e ai loro rapporti con lo Stato centrale. Infine modificava il sistema di elezione del Presidente della Repubblica e la nomina dei giudici della Corte Costituzionale. 


\section{CAMERA DEI DEPUTATI E SENATO: SUPERAMENTO DEL BICAMERALISMO PERFETTO}

La Costituzione italiana prevede un sistema parlamentare composto da due camere, la Camera dei deputati e il Senato. Si tratta di un bicameralismo perfetto o paritario: le due camere svolgono le medesime funzioni e i suoi membri rappresentano gli stessi interessi, quelli nazionali. In particolare entrambe le Camere danno la fiducia al governo e svolgono in maniera condivisa la funzione legislativa, come stabilito dall'art. 70. Gli unici elementi distintivi tra le due ali del Parlamento sono strutturali, e risiedono nelle modalità di formazione delle due camere. Innanzitutto queste hanno una consistenza numerica differente. La Camera dei deputati è composta da 630 deputati. Nel Senato siedono invece 315 senatori. Inoltre il Presidente della Repubblica può nominare cinque senatori a vita (art. 59 Cost.).

In secondo luogo sono previste età diverse per essere eletti deputati e senatori, rispettivamente di 25 e 40 anni. Sono altresì stabilite età differenti per poter eleggere deputati e senatori: hanno diritto di eleggere i deputati, tutti i cittadini italiani che abbiano raggiunto la maggior età; i senatori sono invece eletti a suffragio universale diretto dagli elettori che abbiano superato il venticinquesimo anno di età (art. 58 comma 1). Entrambi i rami del Parlamento hanno una durata analoga, di cinque anni. Relativamente ai meccanismi di elezione della Camera dei Deputati e del Senato, le diverse leggi elettorali hanno sempre adottato regole differenti tra $l^{\prime}$ 'elezione delle due camere, fondandole in particolare sull'art. 57, comma 1, Cost., in base al quale in Senato l'attribuzione dei seggi avviene su base regionale. In tal modo avrebbe dovuto determinarsi una diversa composizione tra i due rami del Parlamento. Tuttavia le soluzioni adottate non portarono ad una diversificazione strutturale tra le due camere, ma ne determinarono una sostanziale corrispondenza. La legge di revisione costituzionale n. 88 del 15 aprile 2016 si proponeva in particolare di superare il bicameralismo perfetto e di differenziare la struttura e le funzioni del Senato. La riforma costituzionale attribuiva al Senato la funzione di rappresentanza degli enti territoriali, nonché di raccordo tra lo Stato e gli altri enti costitutivi della Repubblica. Il numero di senatori veniva ridotto da trecentoquindici a cento: novantacinque rappresentativi degli enti territoriali e cinque di nomina presidenziale.

Mutavano inoltre le modalità di elezione del Senato, che non si sarebbero più svolte a suffragio universale e diretto, ma con un 'elezione di secondo grado da parte delle assemblee degli enti territoriali. In particolare, i novantacinque senatori sarebbero stati eletti con metodo proporzionale dai consigli regionali e dalle province autonome di Trento e Bolzano tra i propri membri e uno tra i sindaci dei comuni del proprio territorio. 
I restanti cinque senatori venivano nominati dal Presidente della Repubblica tra coloro che si distinguevano per meriti nel campo scientifico, artistico, letterario e sociale. Essi duravano in carica sette anni e non potevano essere nuovamente nominati. Rimaneva invece immutata la carica a senatore a vita per gli ex Presidenti della Repubblica. Il Senato diveniva un organo a rinnovo parziale, non sottoposto a scioglimento, poiché la durata dei senatori eletti coincideva con quella degli organi delle istituzioni territoriali dai quali erano stati eletti.

Ogni regione doveva avere almeno due senatori, le province autonome ne avevano due a testa. La ripartizione dei seggi veniva fatta in base alla popolazione risultante dall 'ultimo censimento. Inoltre si prevedeva che i senatori non ricevessero più l'indennità per lo svolgimento del loro incarico di parlamentari, dato che percepivano già il compenso per i ruoli istituzionali rivestiti all'interno degli enti locali.

Era comunque delegata alla legge tutta la normativa di attuazione del sistema come ad esempio la normativa in materia di sostituzione dei senatori in caso di cessazione della carica elettiva regionale o locale. Invece, la struttura della Camera bassa, la sua elezione a suffragio universale diretto e la sua durata non venivano modificate dalla legge di revisione costituzionale 88/ 2016.

In definitiva la riforma realizzava un bicameralismo differenziato. La Camera dei deputati avrebbe dovuto rappresentare la nazione ed essere esclusiva titolare del rapporto fiduciario con il Governo, nonché effettiva titolare della funzione legislativa e della funzione di controllo dell 'operato del Governo. In tal modo si voleva assicurare una maggiore stabilità dell'esecutivo, che infatti non doveva più ottenere la fiducia del Senato, ma soltanto della Camera.

Il Senato invece avrebbe dovuto rappresentare gli enti territoriali e svolgere la funzione di raccordo tra lo Stato, gli altri enti costitutivi della Repubblica e l'Unione europea. Inoltre concorreva con l'altra Camera nell'esercizio del potere legislativo esclusivamente nei casi e secondo le modalità stabilite dal nuovo art. 70, comma 1 della Costituzione. Nei restanti casi le leggi venivano approvate soltanto dalla Camera dei deputati con un procedimento legislativo monocamerale (art. 70 comma 2). Il Senato effettuava poi la valutazione delle politiche pubbliche e dell 'attività delle pubbliche amministrazioni.

Si evidenzia quindi che il bicameralismo perfetto veniva superato attraverso la fine della parità legislativa tra le due Camere e il mantenimento della funzione legislativa piena soltanto in capo alla Camera dei deputati.

La legge di revisione costituzionale introduceva inoltre il comma 2 all'art. 64 Cost., il quale stabiliva che il regolamento della camera disciplinasse lo statuto delle opposizioni, con la finalità di tutelare le minoranze parlamentari e controbilanciare il ruolo della maggioranza. 
Infine la riforma introduceva in Costituzione le pari opportunità nell'accesso agli incarichi parlamentari, prevedendo che fosse promosso un equilibrio di rappresentanza tra donne e uomini da parte delle leggi che stabiliscono le modalità di elezione delle Camere.

\section{POTERI E FUNZIONI LEGISLATIVE: FORMAZIONE DELLE LEGGI}

L'obiettivo principale della riforma costituzionale consisteva nel superamento del bicameralismo perfetto. Questo risultato veniva soprattutto conseguito attraverso la formulazione del nuovo art. 70. Il bicameralismo paritario veniva infatti superato principalmente attraverso la fine della parità legislativa tra le due Camere e il mantenimento della funzione legislativa piena soltanto in capo alla Camera dei Deputati. Infatti, secondo il procedimento legislativo ordinario previsto dal riformato art. 70 comma 2, le leggi statali dovevano essere approvate soltanto dalla Camera dei deputati. Avrebbe invece rappresentato un "eccezione alla regola generale il procedimento legislativo bicamerale, alla luce del quale entrambi i rami del Parlamento avrebbero partecipato al procedimento legislativo con gli stessi poteri, nei casi espressamente identificati dal nuovo comma 1 dell'art. 70.

In base al procedimento legislativo ordinario previsto dalla riforma, la $\mathrm{Ca}$ mera dei deputati aveva un ruolo decisivo nell'approvazione delle leggi. Il Senato invece aveva una mera facoltà di intervento nel procedimento legislativo. La proposta di legge ordinaria doveva infatti essere presentata alla Camera dei deputati e da questa esaminata. In caso di approvazione del testo, questo avrebbe dovuto essere trasmesso al Senato. A questo punto, il Senato aveva la facoltà di esaminare il testo qualora, entro dieci giorni dalla sua trasmissione, ne avesse fatto richiesta un terzo dei suoi componenti. In tal caso, nei successivi trenta giorni il Senato avrebbe potuto deliberare proposte di modificazione del testo esaminato, probabilmente in forma di emendamenti a maggioranza semplice. Il testo sarebbe quindi tornato alla Camera che avrebbe discusso sulle proposte di modificazione votate dal Senato, e avrebbe poi deliberato in via definitiva. Sarebbe spettato al regolamento della Camera stabilire relativamente alle modalità di deliberazione definitiva. In seguito alla deliberazione definitiva, la legge sarebbe stata trasmessa al Presidente della Repubblica per essere promulgata. Tale circostanza si sarebbe verificata anche nel caso in cui il Senato non avesse proceduto all'esame della legge o non avesse deliberato nei termini previsti. Il procedimento ordinario subiva una deroga nei due particolari casi previsti dai commi 4 e 5 dell'art. 70. 
Il primo dei due particolari procedimenti riguardava le leggi che davano attuazione alla "clausola di supremazia" prevista dal nuovo art. 117 comma 4 della Costituzione. In base alla "clausola di supremazia" lo Stato avrebbe potuto esercitare la potestà legislativa nelle materie di competenza regionale, quando ciò fosse risultato necessario per la tutela dell'unità giuridica o economica della Repubblica o la tutela dell'interesse nazionale.

In questo caso il Senato avrebbe potuto disporre l'esame del testo entro dieci giorni dalla data di trasmissione da parte della Camera, dopodiché avrebbe potuto proporre delle modificazioni entro trenta giorni a maggioranza assoluta dei suoi componenti. La Camera dei deputati avrebbe potuto rifiutare questi emendamenti nella votazione finale, soltanto con la maggioranza assoluta dei propri componenti; in caso contrario avrebbe dovuto conformarsi alle modificazioni deliberate dal Senato. Il maggior rilievo attribuito al Senato in questa circostanza, era giustificato dal suo ruolo di garante del sistema delle autonomie locali nell'ottica della riforma.

La seconda variante del procedimento legislativo ordinario era prevista dal comma 5 dell' art. 70 del testo riformato. Questo stabiliva una procedura ordinaria con prevalenza della Camera dei deputati, ma con intervento obbligatorio del Senato in materia di approvazione con legge del bilancio e del rendiconto consuntivo. Infatti, i disegni di legge approvati dalla Camera in questa materia, dovevano essere in ogni caso esaminati dal Senato, senza che ciò venisse richiesto da un certo numero dei suoi componenti. Il Senato aveva tuttavia a disposizione soltanto di quindici giorni dalla data di trasmissione, per deliberare proposte di modifica.

La Camera avrebbe dovuto poi deliberare in via definitiva sulla proposta di modifica presumibilmente con maggioranza semplice, non essendo prescritto alcunché in proposito².

\section{IL PRESIDENTE DELLA REPUBBLICA}

La Costituzione italiana stabilisce che il Presidente della Repubblica venga eletto da parte di un collegio composto da 630 deputati, 315 senatori, i senatori a vita e 58 consiglieri regionali, e che abbia luogo per scrutinio segreto a maggioranza dei due terzi del suddetto collegio. Dopo il terzo scrutinio è sufficiente la maggioranza assoluta. Con le modifiche introdotte dalla riforma costituzionale all'art. 83 Cost., il collegio eleggente era composto esclusivamente dai 730

\footnotetext{
2 Vedi P. Pombeni, La questione costituzionale in Italia, Rzym 2016.
} 
membri del Parlamento (630 deputati e 100 senatori) oltre agli ex presidenti della Repubblica. I delegati regionali non erano più contemplati, essendo rappresentati esclusivamente in Senato.

Inoltre cambiavano le maggioranze necessarie per l'elezione: per i primi tre scrutini era previsto sempre il medesimo quorum di due terzi del collegio, mentre dal quarto era invece richiesto un quorum di tre quinti del collegio stesso. Infine, qualora non si fosse ancora addivenuti all'elezione del Presidente, dal settimo scrutinio sarebbe stata sufficiente la maggioranza dei tre quinti dei votanti. I sostenitori della riforma hanno sottolineato che, grazie alla previsione che stabiliva il quorum nei tre quinti, nessuna forza politica avrebbe presumibilmente potuto eleggere autonomamente il Presidente della Repubblica.

Gli oppositori hanno tuttavia evidenziato che, in virtù dell 'ampio premio di maggioranza di 354 deputati, stabilito dalla legge elettorale vigente in quel momento, il partito di maggioranza, dalla settima votazione, avrebbe potuto comunque determinare l'elezione del Presidente della Repubblica, con l'aggiunta di soli dodici o tredici voti.

\section{IL TITOLO V: REGIONI E ALTRI ENTI LOCALI}

Il titolo V della parte II della Costituzione italiana è relativo agli enti locali (Comuni, Province e Regioni) e alla suddivisione di funzioni e poteri tra questi e lo Stato centrale. Con la riforma costituzionale del 2001, lo Stato italiano assume una struttura più decentrata, tipica degli Stati regionali: alle Regioni vengono attribuite sempre più competenze e una maggior autonomia in campo finanziario e organizzativo. Inoltre viene aumentata l'autonomia regionale in campo legislativo. A tal proposito, 1'art. 117 conseguito alla riforma, effettua innanzitutto, al secondo comma, un elenco di materie su cui lo Stato ha potestà legislativa esclusiva, mentre al comma 3 indica le materie su cui le Regioni hanno una potestà legislativa concorrete a quella statale. In base alla "potestà concorrente", la legislazione dello Stato determina i principi fondamentali della materia, mentre il resto della disciplina, quella di dettaglio, compete alla Regione, la quale deve ovviamente attenersi alle direttive fornite dallo Stato. Infine, alla luce della clausola residuale (comma 4), tutte le materie non comprese tra quelle di competenza statale o concorrente, spettano alla competenza esclusiva delle Regioni.

Con la riforma costituzionale del 2016, si stabiliva un 'inversione di tendenza rispetto all'indirizzo perseguito nel 2001, con un sostanziale accentramento delle funzioni in capo allo Stato. Infatti, il nuovo art. 117 Cost. ampliava notevolmente la potestà legislativa dello Stato, eliminando invece totalmente quella 
concorrente. Le materie rientranti in quest'ultima venivano dunque in maggior parte assegnate alla potestà legislativa esclusiva dello Stato, prevista dal comma 2. Venivano invece attribuite in via esclusiva alle Regioni, oltre alle materie espressamente elencate nel nuovo comma 4, anche quelle non espressamente riservate alla competenza esclusiva dello Stato, in virtù della clausola residuale.

I poteri dello Stato venivano tuttavia ulteriormente rafforzati con la previsione della clausola di supremazia, in base alla quale la legge dello Stato poteva intervenire in materie riservate alla competenza regionale quando lo richiedesse la tutela dell'unità giuridica o economica della Repubblica, ovvero la tutela dell'interesse nazionale.

In secondo luogo, con la riforma costituzionale del 2016, venivano anche abolite le Province. Inoltre veniva modificato l'art. 116 sul cosiddetto "federalismo differenziato": la concessione di una maggiore autonomia alle singole Regioni, in materie attribuite alla competenza statale, sarebbe stata possibile non più semplicemente con legge statale su iniziativa delle stesse, dovendo la Regione richiedente necessariamente trovarsi in condizione di equilibrio tra entrate e le spese del proprio bilancio.

\section{CORTE COSTITUZIONALE}

La riforma del 2016 introduceva alcune modifiche sulle nomine dei giudici della Corte Costituzionale e affidava un'ulteriore competenza alla medesima. Ai sensi dell'art. 135 Cost., i giudici della Corte Costituzionale sono quindici, nominati per un terzo dal Presidente della Repubblica, per un terzo dalla suprema magistratura ordinaria ed amministrativa, e per un terzo dal Parlamento in Seduta Comune. Qualora la riforma costituzionale del 2016 fosse entrata in vigore, i giudici della Consulta sarebbero rimasti quindici e nominati per un terzo dal Presidente della Repubblica, per un terzo dalla suprema magistratura ordinaria e amministrativa; tuttavia, in virtù delle riforme strutturali del Parlamento, per il restante terzo, la nomina sarebbe stata effettuata disgiuntamente da Camera e Senato: pertanto tre giudici sarebbero stati nominati dalla Camera dei deputati e due dal Senato. Questi sarebbero stati eletti da ciascuna camera a scrutinio segreto e con la maggioranza dei due terzi dei propri componenti. Dal terzo scrutinio sarebbe stata sufficiente la maggioranza dei tre quinti dei componenti di ciascuna camera.

In secondo luogo, alla Corte Costituzionale veniva attribuita la funzione di controllo preventivo della legittimità costituzionale delle leggi che disciplinano l'elezione dei membri della Camera dei deputati e del Senato, in caso di ricorso 
motivato presentato da almeno un quarto dei componenti della Camera dei deputati o da almeno un terzo dei componenti del Senato, entro dieci giorni dall 'approvazione della legge stessa.

La Corte Costituzionale avrebbe dovuto pronunciarsi sul ricorso entro il termine di trenta giorni, mantenendo sospeso il termine per la promulgazione della legge fino alla pronuncia. In caso di dichiarazione di illegittimità costituzionale, la legge non sarebbe stata promulgata.

\section{CNEL}

La riforma costituzionale del 2016 prevedeva l'abolizione del Cnel disciplinato dall ,art. 99 Cost. Il Cnel, Consiglio nazionale dell'economia e del lavoro, è composto da 65 membri nominati con decreto presidenziale previa deliberazione del Consiglio dei Ministri e dura in carica cinque anni. I suoi membri sono esperti e rappresentanti di diverse categorie produttive.

Ha la funzione di consulenza nei confronti di Governo e Camere in materia di economia e di lavoro, inoltre ha poteri di iniziativa legislativa in materia economico-sociale. Era stato previsto dai Costituenti con la finalità di integrare la rappresentanza politica con una rappresentanza diretta di interessi economicosociali, per fronteggiare le difficoltà della prima sul versante del raccordo con la società. Tuttavia il Consiglio nazionale dell'economia e del lavoro ha riscosso uno scarso successo, a causa della difficoltà a rappresentare la dinamica complessità sociale e il mutevole peso degli interessi dei diversi gruppi socio-economici. Inoltre questi ultimi, solitamente tendono a relazionarsi e negoziare direttamente con gli organi costituzionali, senza ricorrere ad altre forme di mediazione. Per questi motivi, la riforma costituzionale del 2016 ne proponeva l'abolizione, abrogando integralmente 1 'art. 99 Cost.

\section{CONCLUSIONI}

Una prima evidente criticità era la scarsa condivisione della proposta di riforma, appoggiata soltanto da una parte del partito di maggioranza: una riforma così vasta e incidente sull'intero assetto costituzionale avrebbe necessitato di una condivisione molto più ampia. Una seconda perplessità che è stata espressa riguardo a questa riforma costituzionale è proprio quella relativa all'ampiezza delle modifiche. Queste vanno dal superamento del bicameralismo perfetto, obiettivo primario e centrale della legge di revisione costituzionale, a tutta una 
serie di altri interventi incidenti sulle altre istituzioni e organi strutturali della Repubblica in un 'ottica di ricerca dell 'equilibrio e del coordinamento istituzionale, nonché al fine di ottenere un sistema maggiormente semplificato ed efficiente. Tuttavia il risultato conseguitone era un sistema eccessivamente semplificato e squilibrato a favore degli organi statali e in particolare dell'esecutivo.

Le contromisure adottate in termini di bilanciamento non risultavano adeguate e la logica dell'efficienza induceva il legislatore ad ipotizzare un sistema costituzionale che perseguiva l'obiettivo primario di pervenire a decisioni rapide, immediate, ma sicuramente meno ponderate e pressoché unilaterali. D'altronde, i principi della governabilità e della stabilità dell 'esecutivo, indicati come punti centrali dai promotori della riforma, rischiano di svuotare il ruolo del Parlamento come luogo del compromesso politico, nonché la centralità costituzionale che assume nel nostro che questo assume nel nostro questo assume nel nostro sistema costituzionale.

\section{BIBLIOGRAFIA:}

Occhetta F., La riforma della Costituzione, www.stefanoceccanti.wordpress.com. (12.06.2017).

Pombeni P., La questione costituzionale in Italia, Roma 2016. 